\title{
EFEITO DO INIBIDOR DE NITRIFICAÇÃO «AM» EM ALGUNS SOLOS DO ESTADO DE SÃO PAULO*
}

\author{
A. M. L. Neptune** \\ S. M. TSAI SAITo $* * *$
}

\section{RESUMO}

Utilizou-se o inibidor de nitrificação "AM" em quatro solos diferentes do Estado de São Paulo, a fim de se determinar a dose efetiva de inibição da nitrificação em cada solo. As doses usadas $(0,12,24,36,48$ e $60 \mathrm{ppm})$ de "AM" não foram suficientes para atuarem significativamente no Latossol Roxo e no Podzolizado de Lins e Marília, var. Marília; porém resultados positivos foram obtidos com os solos Podzolizado de Piracicaba e Regossol Intergrade, para os quais, doses de 24 a 60 ppm foram suficientes para atuarem por um período de 60 dias.

\section{INTRODUÇÃO}

Entre os inibidores, destacam-se o "N-Serve" (2-cloro-6-triclorometil piridina) e o "AM" (2-amino-4-cloro-6-metil pirimidina). Por ser o primeiro mais efetivo, maior volume de trabalhos foram realizados com ele. Ambos estão sendo largamente usados para inibição da nitrificação em solos alagados ou secos, e em diversas culturas tais como milho, arroz, cana-de-açúcar, trigo, etc., com uma considerável economia de fertilizante e aumento de produção (DAS, 1965; PRASAD et al., 1966; PRASAD, 1968; GASSER et al., 1967; GASSER e IORDANOU, 1967; LAKHADIVE e PRASAD, 1970).

Usando "AM" em solos tropicais, WEIR e DAVIDSON, 1968, obtiveram 25\% de aumento de produção da grama Pangola, além de um aumento de $60 \%$ na eficiência do fertilizante.

\footnotetext{
* Trabalho realizado em parte com o auxílio da Comissão Nacional de Energia Nuclear. Entregue para publicação em 30.12.76.

* Departamento de Solos e Geologia e Divisão de Ciências do Solo do CENA ESALQ, USP.

** Pesquisadora do Centro de Energia Nuclear na Agricultura (CENA), ESALQ USP.
} 
O efeito do inibidor depende das características do solo: capacidade de troca catiônica, teor de argila, área superficial dos solos (WEIR, 1969).

O problema maior é determinar a concentração mínima efetiva que não seja fitotóxica. No caso de "AM", a dosagem recomendada por PATRICK et al., 1968, foi de 2-5 ppm para solo barro-arenoso. RAJALE e PRASAD, 1970, utilizaram 5-10 ppm de "AM".

O presente experimento foi realizado em laboratório, com a finalidade de determinar a dose mínima efetiva do inibidor "AM" em diferentes solos, uma vez que existem poucas informações a respeito de concentrações de "AM" em solos.

\section{MATERIAIS E MÉTODOS}

Os solos Regossol Intergrade (R), Latossol Roxo (LR), Podzolizado de Lins e Marília (Pml) e Podzolizado de Piracicaba (Pvp) foram usados, correspondendo a $60 \%$ dos solos do Estado de São Paulo. Algumas características químicas e granulométricas estão descritas no Quadro 1.

Quadro 1 - Características químicas e granulométricas dos solos Regossol Intergrade (R); Latossol Roxo (LR); Podzolizado de Lins e Marília, var. Marília (Pml) e Podzolizado de Piracicaba (Pvp).

\begin{tabular}{|c|c|c|c|c|c|c|c|c|c|c|}
\hline & ${ }_{1} \mathrm{H}$ & M.O. & $\begin{array}{l}(\mathrm{emg}) \\
\mathrm{Ca}^{2+}\end{array}$ & $\begin{array}{l}100 \mathrm{~g} \\
\mathrm{Mg}^{2+}\end{array}$ & $\begin{array}{l}\text { solo) } \\
\mathrm{K}^{1+}\end{array}$ & $\begin{array}{l}\text { Areia } \\
\text { Crrossa } \\
\%\end{array}$ & $\begin{array}{c}\text { Areia } \\
\text { Fina } \\
\%\end{array}$ & $\begin{array}{l}\text { Silte } \\
\%\end{array}$ & Arg. & Clas. Text. \\
\hline $\mathrm{R}$ & 5.5 & 1.19 & 1.56 & 0.44 & 0.06 & 33.3 & 57.2 & 1.7 & 7.8 & areia \\
\hline LR & 5.3 & 3.07 & 2.04 & 1.21 & 0.15 & 8.2 & 25.5 & 13.3 & 53.0 & argila \\
\hline Pml & 4.7 & 0.67 & 3.01 & 0.23 & 0.05 & 23.6 & 66.7 & 3.5 & 6.2 & areia \\
\hline$P_{v p}$ & 5.5 & 1.21 & 3.03 & 0.63 & 0.21 & 47.1 & 9.9 & 31.9 & 11.1 & areia \\
\hline
\end{tabular}

Os tratamentos para cada solo foram:

Tratamento 1: $\quad\left(\mathrm{NH}_{4}\right)_{2} \mathrm{SO}_{+}(48 \mathrm{ppm})$

Tratamento 2: $\mathrm{A}+6 \mathrm{ppm}$ "AM"

Tratamento 3: A + 12 ppm "AM"

Tratamento 4: A + 24 ppm "AM" 
$\begin{array}{ll}\text { Tratamento 5: } & \mathrm{A}+36 \mathrm{ppm} \text { "AM" } \\ \text { Tratamento 6: } & \mathrm{A}+48 \mathrm{ppm} \text { "AM" } \\ \text { Tratamento 7: } & \mathrm{A}+60 \mathrm{ppm} \text { "AM" }\end{array}$

Amostras com $100 \mathrm{~g}$ de solo foram incubadas aerobicamente em frascos de vidro de $200 \mathrm{ml}$ de volume, com 3 repetições para cada tratamento. A umidade foi mantida a $75 \%$ da máxima retenção de umidade e a temperatura média variou ao redor de $25^{\circ} \mathrm{C}$, durante o decorrer de todo o experimento.

Análises periódicas foram realizadas aos 15, 30 e 60 dias de incubação, quanto aos teores de $\mathrm{NH}_{4}{ }^{+}$e $\mathrm{NO}_{3}^{-}$, segundo o método de BREMNER, 1965.

\section{RESULTADOS E DISCUSSÃO}

\section{Efeitos dos Solos}

Devido à grande influência das características do solo (WEIR, 1969), o efeito do inibidor não foi igual nos quatro solos utilizados. As doses efetivas foram mais elevadas que os utilizados por outros autores (PATRICK et al., 1968 e RAJALE e PRASAD, 1970).

O efeito do "AM" foi menor no Latossol Roxo, onde o nitrogênio amoniacal adicionado apresentou uma taxa de mineralização maior que

Quadro 2 - Teor de $\mathrm{NH}^{+}{ }_{4}$ e $\mathrm{NO}^{-}{ }_{3}$ (ppm) apresentados pelos solos nos períodos de incubação $(15,30$ e 60 dias $)$.

\begin{tabular}{lcrrrr}
\hline & Tempo & $\mathrm{R}$ & LR & Pml & Pvp \\
\hline \hline & $15 \mathrm{~d}$ & $21,29 \mathrm{c}$ & $78,06 \mathrm{a}$ & $20,92 \mathrm{c}$ & $35,10 \mathrm{~b}$ \\
$\mathrm{NH}^{+}{ }_{4}$ & $30 \mathrm{~d}$ & $22,38 \mathrm{c}$ & $49,92 \mathrm{a}$ & $23,05 \mathrm{c}$ & $43,18 \mathrm{~b}$ \\
& $60 \mathrm{~d}$ & $19,52 \mathrm{c}$ & $30,82 \mathrm{~b}$ & $20,98 \mathrm{c}$ & $41,88 \mathrm{a}$ \\
& $15 \mathrm{~d}$ & $9,33 \mathrm{~b}$ & $15,07 \mathrm{a}$ & $8,32 \mathrm{c}$ & $10,12 \mathrm{~b}$ \\
$\mathrm{NO}^{-}$ & $30 \mathrm{~d}$ & $12,77 \mathrm{~b}$ & $30,15 \mathrm{a}$ & $11,42 \mathrm{~b}$ & $10,10 \mathrm{c}$ \\
& $60 \mathrm{~d}$ & $26,65 \mathrm{~b}$ & $43,92 \mathrm{a}$ & $14,87 \mathrm{c}$ & $13,52 \mathrm{c}$ \\
\hline
\end{tabular}

* Teste $\mathrm{t}$ : tratamentos com letras semelhantes não diferem significativamente $(\mathrm{P}<5 \%)$ para cada período de incubação. 
os demais solos. A presença de alto teor de argila neste solo pode ter contribuído para esse resultado.

O solo Podzolizado de Piracicaba tratado com "AM" apresentou alto teor de $\mathrm{NH}_{4}{ }^{+}$e baixo teor de $\mathrm{NO}_{3}{ }^{-}$em relação à testemunha, indicando efeito inibidor neste solo.

A influência do "AM" nos dois solos restantes, Regossol Intergrade e Podzolizado de Lins e Marília, var. Marília, se fez também presente.

Os teores de $\mathrm{NH}_{4}{ }^{+}$dos solos tratados se mantiveram quase constantes durante o experimento e os teores de $\mathrm{N}$ total sofreram acréscimo com o tempo.

Os dados estão apresentados no Quadro 2.

\section{Efeitos dos tratamentos nos 4 solos}

Em geral, todos os tratamentos foram superiores à testemunha (Quadro 3). A partir de $36 \mathrm{pp}$ mde "AM", o teor de $\mathrm{NH}_{+}^{+}$é mantido por um período de 60 dias, assim como há um ligeiro acréscimo no teor de $\mathrm{N}$ total em todos os solos tratados.

Quadro 3 - Efeito dos tratamentos nos teores de $\mathrm{NH}^{+}{ }_{4} \mathrm{e} \mathrm{NO}_{3}^{-}$(ppm) nos solos e nos diferentes tempos de incubação $(15,30$ e 60 dias $)$.

\begin{tabular}{|c|c|c|c|c|c|c|c|c|}
\hline & Tempo & 1 (Test) & 2 & 3 & 4 & 5 & 6 & 7 \\
\hline & $15 \mathrm{~d}$ & $31,69 c$ & $39,99 \mathrm{ab}$ & $41,27 \mathrm{a}$ & $36,62 b$ & $40,83 \mathrm{ab}$ & $42,58 \mathrm{a}$ & $38,92 \mathrm{ab}$ \\
\hline \multirow[t]{3}{*}{$\mathrm{NH}^{+}{ }_{4}$} & $30 \mathrm{~d}$ & $27,80 \mathrm{c}$ & $36,11 \mathrm{~h}$ & $34,56 b$ & $35,12 b$ & $33,22 b$ & $35,35 b$ & $40,28 \mathrm{a}$ \\
\hline & $60 d$ & $23,33 \mathrm{c}$ & $25,61 \mathrm{~b}$ & $28,29 \mathrm{ab}$ & $27,84 b$ & $31,12 \mathrm{ab}$ & $31,27 \mathrm{a}$ & $30,65 a b$ \\
\hline & $15 \mathrm{~d}$ & $18.52 \mathrm{a}$ & $8,75 \mathrm{c}$ & $9,6.5 \mathrm{bc}$ & $10,09 b$ & $9,19 b c$ & $9,54 b c$ & $9,22 b c$ \\
\hline \multirow[t]{2}{*}{$\mathrm{NO}^{-}{ }_{3}$} & $30 \mathrm{~d}$ & $22,11 \mathrm{a}$ & $16,92 \mathrm{~b}$ & $16,51 b c$ & $14,88 \mathrm{c}$ & $15,52 \mathrm{bc}$ & $14,18 \mathrm{c}$ & $12,66 \mathrm{c}$ \\
\hline & $60 \mathrm{~d}$ & $28,29 a$ & $24,82 b$ & $23,47 \mathrm{~b}$ & $24,94 b$ & $22,92 \mathrm{~b}$ & $23,95 \mathrm{~b}$ & $24,68 b$ \\
\hline
\end{tabular}

* Teste $t$ : tratamentos com letras semelhantes não diferem significativamente $(\mathrm{P}<5 \%)$ para cada período de incubação.

\section{Efeito do “AM" no Regossol Intergrade}

Pelo Quadro 4, e Figuras 1 e 2, observamos que a partir de 24 ppm o efeito do "AM" apresentou diferença significativa com a testemunha, diferença que aumentou com o tempo. 
Quadro 4 - Efeito dos tratamentos nos teores de $\mathrm{NH}_{4}^{+}$e $\mathrm{NO}_{3}^{-}$(ppm) do solo Regossol Intergrade ,nos 3 períodos de incubação.

\begin{tabular}{|c|c|c|c|c|c|c|c|c|}
\hline & Tempo & 1 (Test) & 2 & 3 & 4 & 5 & 6 & 7 \\
\hline $15 \mathrm{~d}$ & $\begin{array}{l}\mathrm{NH}^{+}{ }_{4} \\
\mathrm{NO}^{-}{ }_{3}\end{array}$ & $\begin{array}{l}17,97 \mathrm{c} \\
23,33 \mathrm{a}\end{array}$ & $\begin{array}{c}19,95 \mathrm{bc} \\
7,93 \mathrm{a}\end{array}$ & $\begin{array}{c}20,65 \mathrm{abc} \\
7,12 \mathrm{~b}\end{array}$ & $\begin{array}{r}24,33 \mathrm{a} \\
6,88 \mathrm{~b}\end{array}$ & $\begin{array}{c}22,17 \mathrm{ab} \\
5,95 \mathrm{~b}\end{array}$ & $\begin{array}{c}22,40 \mathrm{ab} \\
8,63 \mathrm{a}\end{array}$ & $\begin{array}{c}21,58 \mathrm{abc} \\
5,48 \mathrm{~b}\end{array}$ \\
\hline $30 \mathrm{~d}$ & $\begin{array}{l}\mathrm{NH}^{+}{ }_{4} \\
\mathrm{NO}^{-}{ }_{3}\end{array}$ & $\begin{array}{r}9,57 \mathrm{~d} \\
30,68 \mathrm{a}\end{array}$ & $\begin{array}{l}24,50 \mathrm{~b} \\
11,78 \mathrm{~b}\end{array}$ & $\begin{array}{l}23,57 \mathrm{~b} \\
10,27 \mathrm{c}\end{array}$ & $\begin{array}{r}25,08 \mathrm{~b} \\
9,89 \mathrm{c}\end{array}$ & $\begin{array}{l}25,32 \mathrm{~b} \\
9,10 \mathrm{~cd}\end{array}$ & $\begin{array}{r}20,77 \mathrm{c} \\
9,92 \mathrm{c}\end{array}$ & $\begin{array}{r}27,88 \mathrm{a} \\
7,82 \mathrm{~d}\end{array}$ \\
\hline $60 \mathrm{~d}$ & $\begin{array}{l}\mathrm{NH}^{+}{ }_{4} \\
\mathrm{NO}^{-}{ }_{3}\end{array}$ & $\begin{array}{l}11,20 \mathrm{~d} \\
35,92 \mathrm{a}\end{array}$ & $\begin{array}{l}17,38 \mathrm{c} \\
25,02 \mathrm{bc}\end{array}$ & $\begin{array}{l}20,65 \mathrm{~b} \\
23,22 \mathrm{~d}\end{array}$ & $\begin{array}{l}22,17 \mathrm{a} \\
23,10 \mathrm{~d}\end{array}$ & $\begin{array}{l}23,10 \mathrm{a} \\
25,32 \mathrm{c}\end{array}$ & $\begin{array}{l}21,70 \mathrm{ab} \\
26,95 \mathrm{~b}\end{array}$ & $\begin{array}{l}20,42 \mathrm{~b} \\
26,02 \mathrm{bc}\end{array}$ \\
\hline
\end{tabular}

* Teste $\mathrm{t}$ : tratamentos com letras semelhantes não diferem significativamente $(\mathrm{P}<5 \%)$ para cada período de incubação.

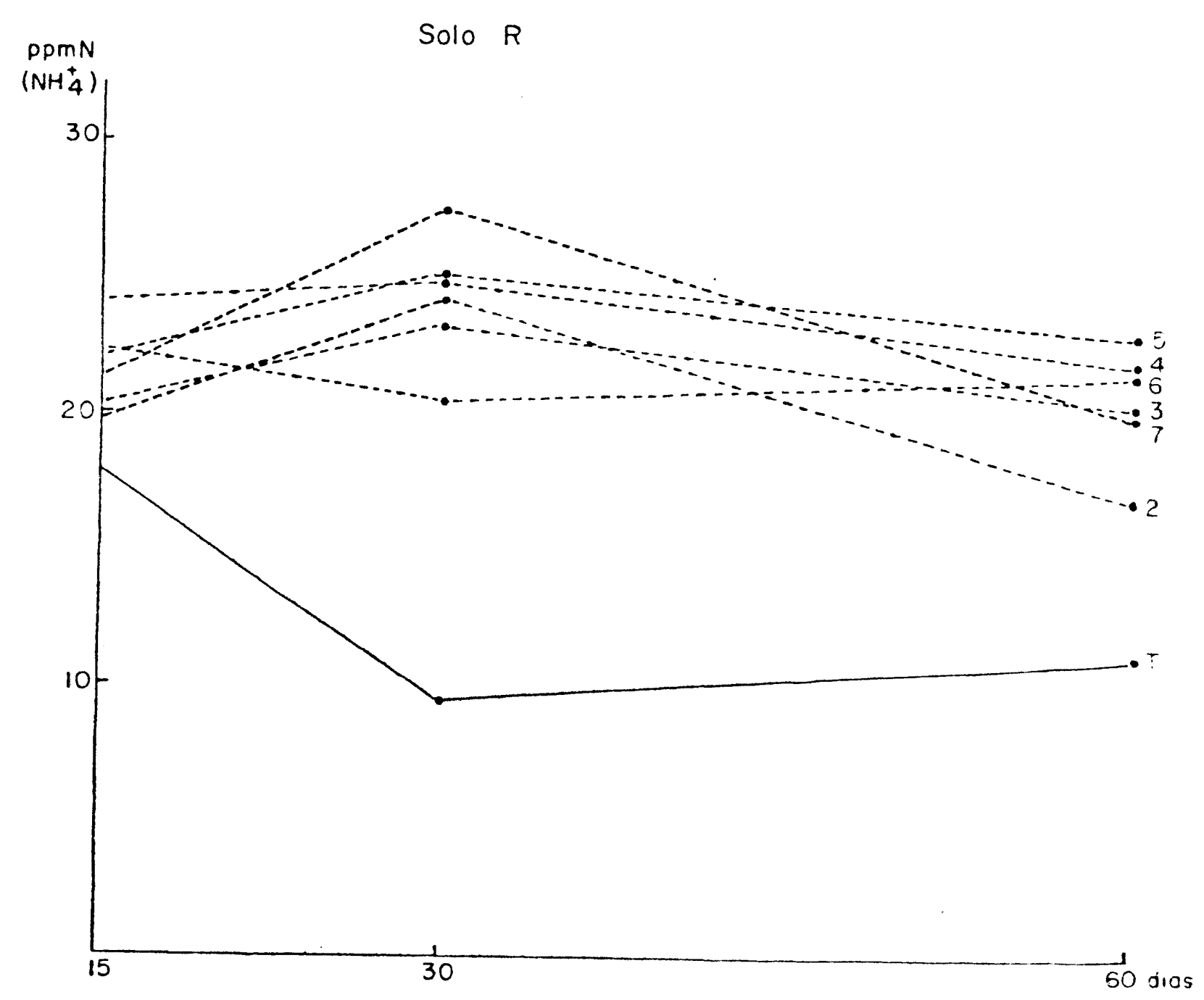

Fig. 1 - Teores de $\mathrm{NH}_{4}^{+}$do solo Regossol nos tres períodos de incubação 


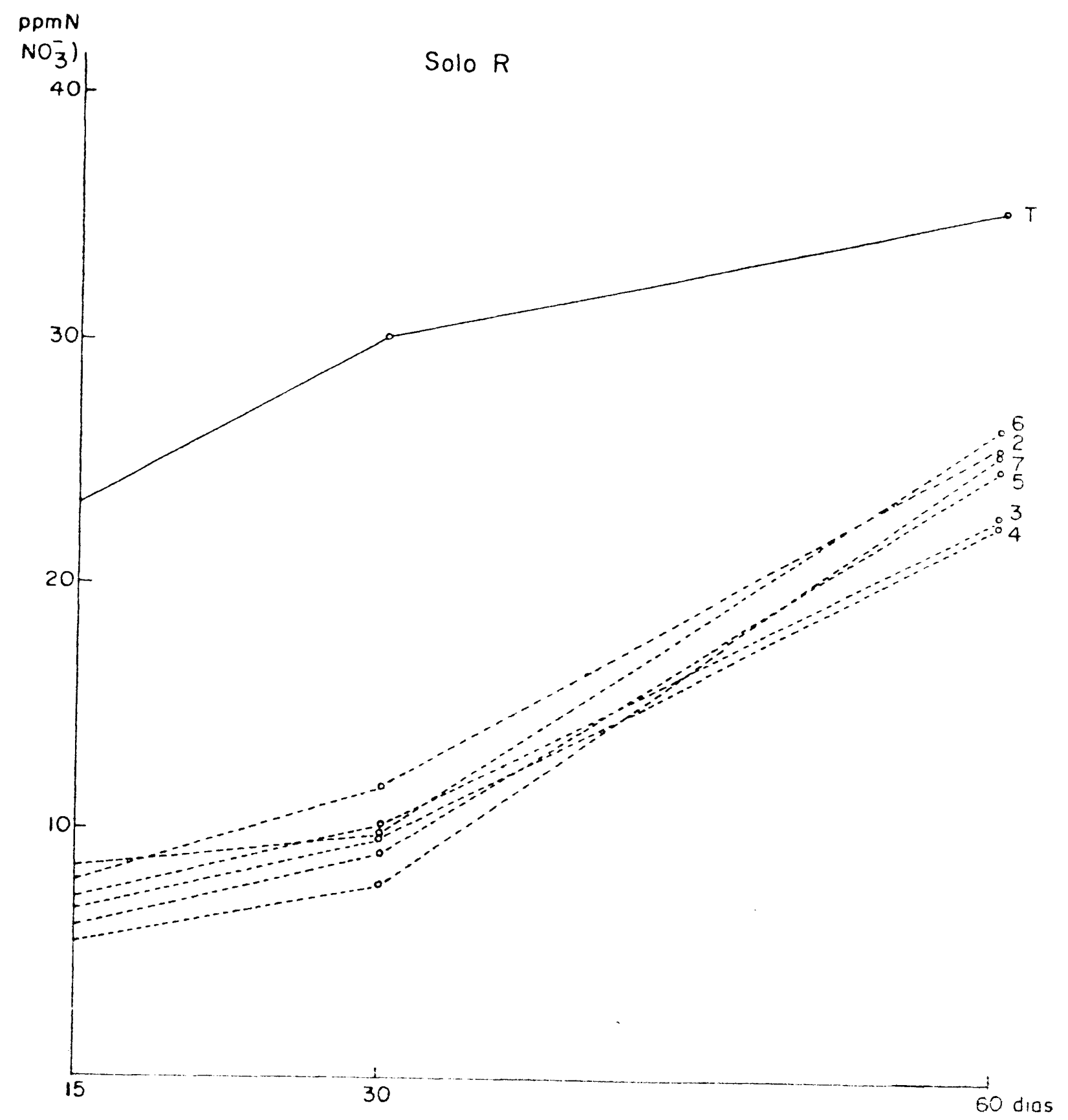

Fig. 2 - Teores de $\mathrm{NO}_{3}^{-}$do solo Regossol nos tres períodos de incubação

Por ser o Regossol Intergrade um solo com baixo teor de argila, recomendamos a dose de 24 ppm de "AM", por um período de 60 dias.

\section{Efeito do "AM" no Latossol Roxo}

O efeito do "AM" não foi significativo nesse solo, apesar dos tratamentos terem apresentado diferenças com a testemunha. Aos 15 dias de incubação, o teor de $\mathrm{NH}_{4}{ }^{+}$foi maior em todos os tratamentos com "AM", mas aos 30 e principalmente aos 60 dias, essa diferença se fez menor em relação à testemunha, indicando a perda do efeito do "AM". 
Doses mais elevadas de "AM" tiveram, em geral, efeito superior às doses menores.

Para se saber realmente a atuação do inibidor neste solo, já que as doses só foram eficientes por curto tempo, estudos deverão ser feitos com doses mais elevadas que as usadas neste experimento e incluindo plantas para observação da fitotoxidade do produto químico.

Os dados obtidos neste solo estão apresentados no Quadro 5 e Figuras 3 e 4 .

Quadro 5 - Efeito dos tratamentos nos teores de $\mathrm{NH}_{+}^{+}$e $\mathrm{NO}_{-}^{-}$(ppm) do solo Latossol Roxo, nos 3 períodos de incubação.

\begin{tabular}{|c|c|c|c|c|c|c|c|c|}
\hline & Tempo & 1 (Test) & 2 & 3 & 4 & 5 & 6 & 7 \\
\hline \multirow{2}{*}{$15 d$} & $\mathrm{NH}^{+}{ }_{4}$ & 58,05 & 84,70 & 88,55 & 66,73 & 85,87 & 89,83 & 72,72 \\
\hline & $\mathrm{NO}^{-}{ }_{3}$ & $30,68 \mathrm{a}$ & $10,62 \mathrm{~b}$ & $14,00 \mathrm{~b}$ & $14,82 \mathrm{~b}$ & $11,78 b$ & $10,97 \mathrm{~b}$ & $12,60 \mathrm{~b}$ \\
\hline \multirow{2}{*}{$30 \mathrm{~d}$} & $\mathrm{NH}_{4}^{+}$ & 38,50 & 52,97 & 48,65 & 49,82 & 42,47 & 54,72 & 62,30 \\
\hline & $\mathrm{NO}^{-}{ }_{3}$ & 33,37 & 31,97 & 32,78 & 29,98 & 32,78 & 26,37 & 23,80 \\
\hline \multirow{2}{*}{$60 \mathrm{~d}$} & $\mathrm{NH}^{+}{ }_{4}$ & 27,18 & 28,47 & 33,48 & 23,62 & 37,22 & 35,00 & 30,80 \\
\hline & $\mathrm{NO}^{-}$ & 44,10 & 43,17 & 41,77 & 48,65 & 40,13 & 42,58 & 47,02 \\
\hline
\end{tabular}

* Teste $\mathrm{t}$ : tratamentos com letras semelhantes não diferem significativamente $(\mathrm{P}<5 \%)$, para cada período de incubação; tratamentos sem letras não diferem significativamente entre si.

Efeito do "AM" no Podzolizado de Lins e Marília, var. Marília

Doses elevadas de "AM" (maiores de $30 \mathrm{ppm}$ ) contribuiram para maiores teores de $\mathrm{NH}_{4}{ }^{+}$nesse solo, apesar de não terem sido significativos.

O inibidor deverá ser testado novamente para esse solo, usando-se doses superiores aos resultados neste experimento e observando a fitotoxidade em plantas.

Os dados obtidos para este solo estão no Quadro 6 e Figuras 5 e 6. 


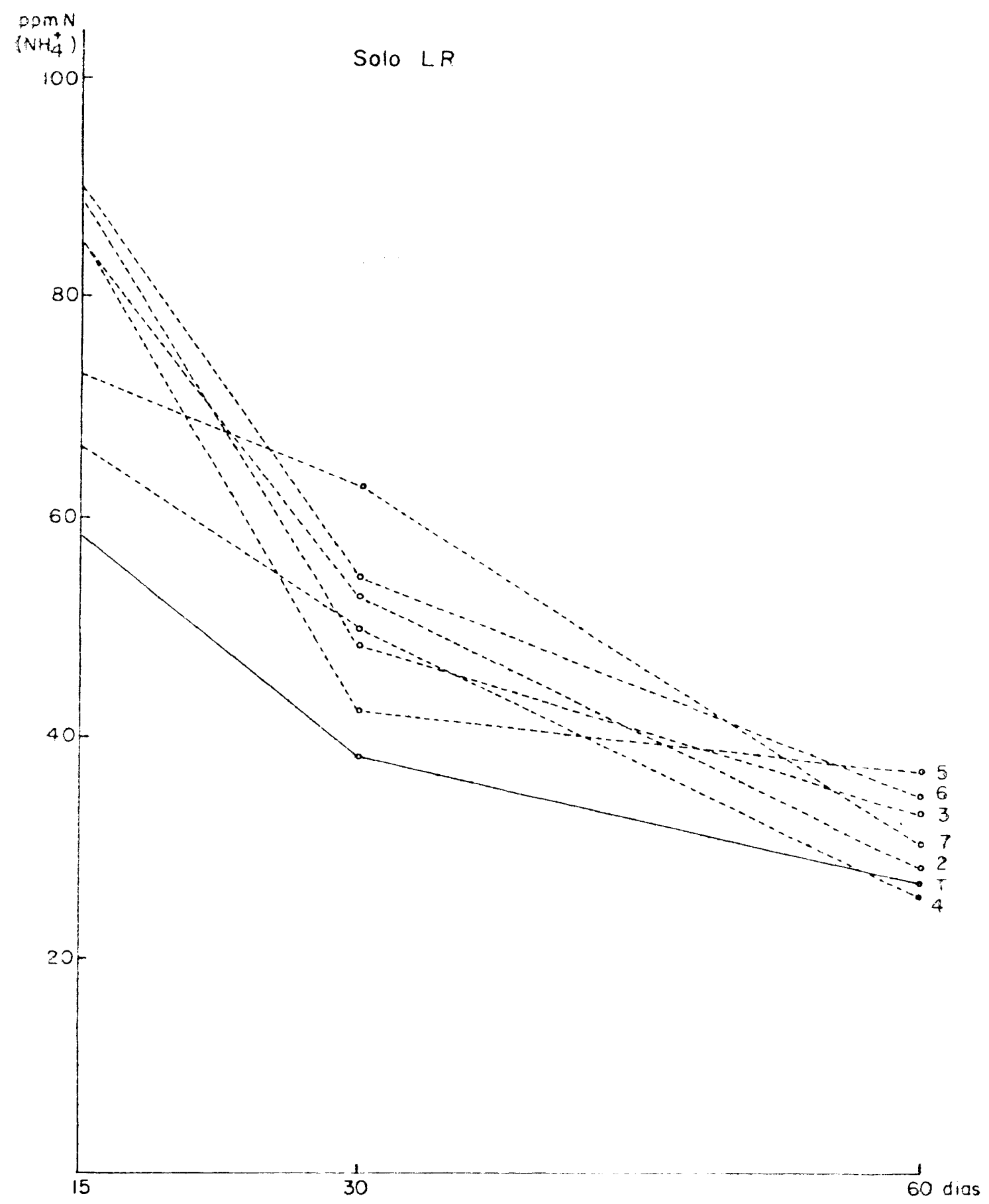

Fig. 3 - Teores de $\mathrm{NH}^{+}{ }_{4}$ do solo Latossol Roxo nos tres períodos de incubação 


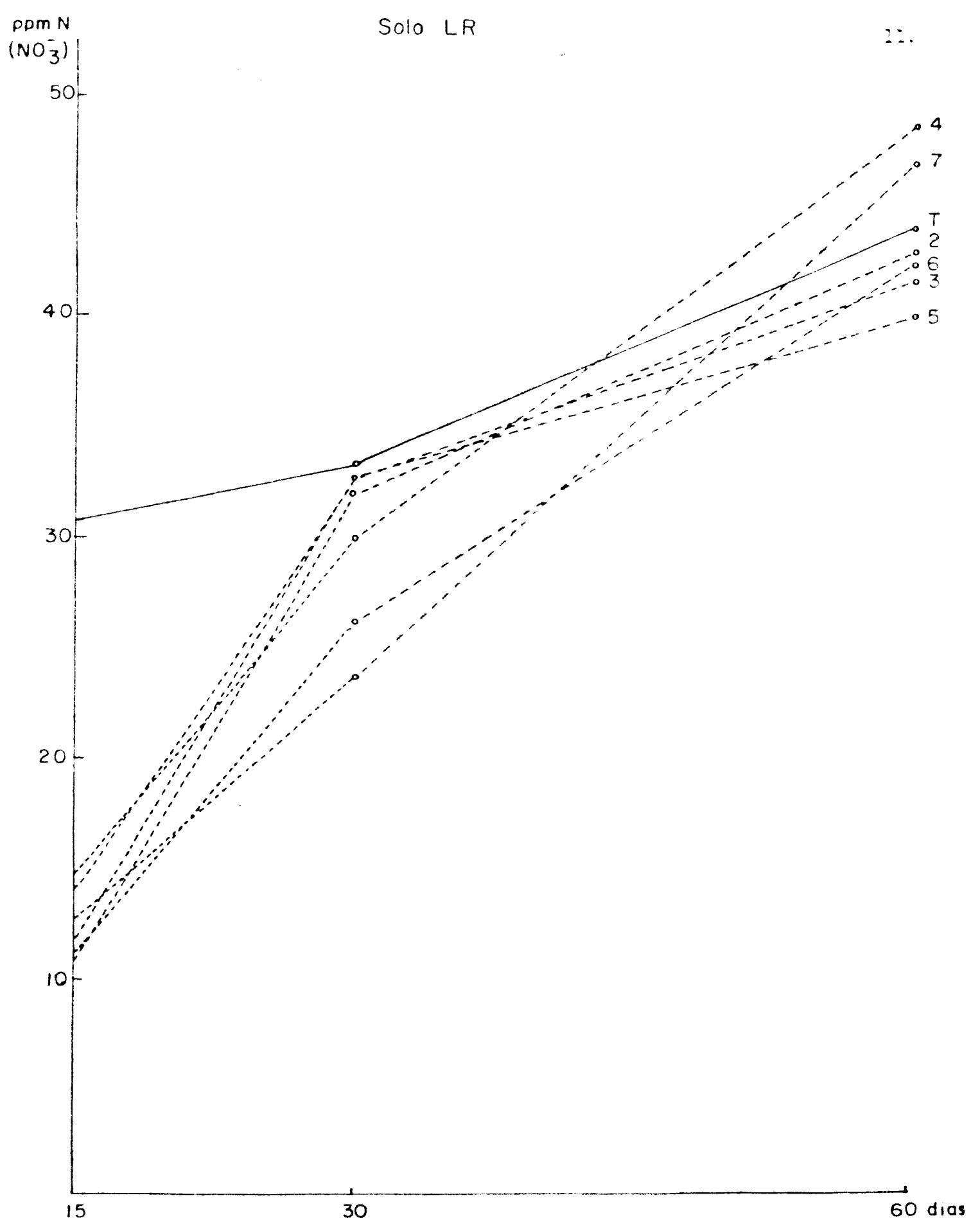

Fig. 4 - Teores de $\mathrm{NO}^{-}$, do solo Latossol Roxo nos tres períodos de incubação 
Quadro 6 - Efeito dos tratamentos nos teores de $\mathrm{NH}_{4}^{+}$e $\mathrm{NO}_{3}^{-}$(ppm) do solo Podzolizado de Lins e Marília, var. Marília, nos 3 períodos de incubação.

\begin{tabular}{|c|c|c|c|c|c|c|c|c|}
\hline & Tempo & 1 (Test) & 2 & 3 & 4 & 5 & 6 & 7 \\
\hline \multirow{2}{*}{$15 \mathrm{~d}$} & $\mathrm{NH}_{4}^{+}$ & 18,55 & 18,90 & 20,30 & 20,53 & 22,40 & 22,40 & 23,33 \\
\hline & $\mathrm{NO}_{3}^{-}$ & 9,57 & 8,05 & 8,40 & 8,28 & 8,17 & 8,17 & 7,58 \\
\hline \multirow{2}{*}{$30 \mathrm{~d}$} & $\mathrm{NH}_{4}^{+}$ & 22,98 & 24,27 & 22,40 & 22,98 & 22,52 & 22,52 & 23,68 \\
\hline & $\mathrm{NO}_{-}^{-}$ & 13,07 & 12,13 & 11,08 & 10,62 & 11,67 & 10,85 & 10,50 \\
\hline \multirow{2}{*}{$60 \mathrm{~d}$} & $\mathrm{NH}_{4}^{+}$ & $16,92 \mathrm{c}$ & $19,37 b c$ & $19,25 \mathrm{bc}$ & $22,63 \mathrm{~b}$ & $22,51 b$ & $22,87 \mathrm{~b}$ & $23,33 a$ \\
\hline & $\mathrm{NO}^{-}$ & $16,92 \mathrm{a}$ & $16,92 \mathrm{a}$ & $16,22 \mathrm{a}$ & $15,40 \mathrm{a}$ & $12,83 \mathrm{~b}$ & $13,53 b$ & $12,25 b$ \\
\hline
\end{tabular}

* Teste $\mathrm{t}$ : tratamentos com letras semelhantes não diferem significativamente $(\mathrm{P}<5 \%)$, para cada período de incubação; tratamentos sem letras não diferem significativamente entre si.

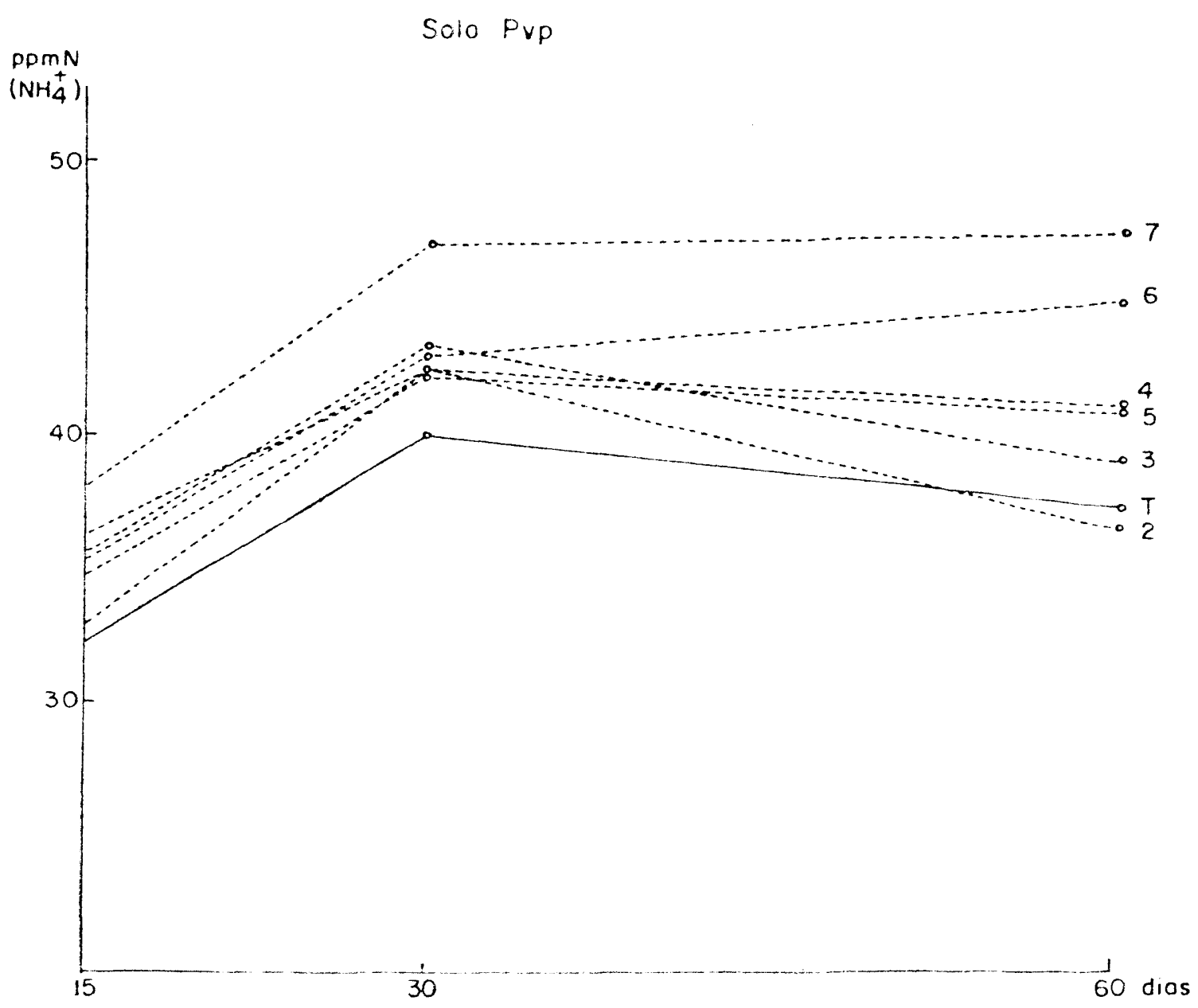

Fig. 5 - Teores de $\mathrm{NH}_{4}{ }_{4}$ do solo Podzolizado de Lins e Marília, var. Marília nos tres períodos de incubação 


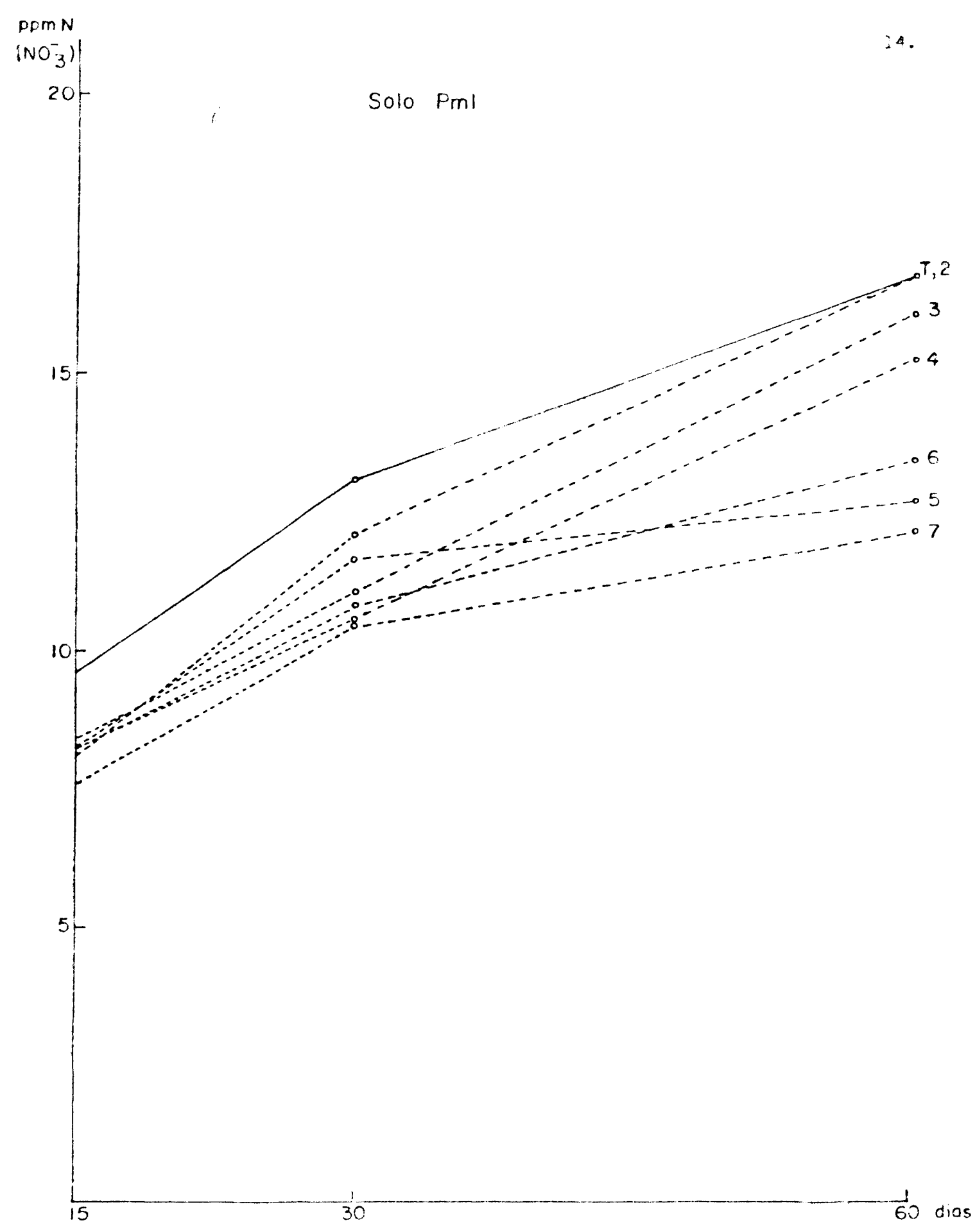

Fig. 6 - Teores de $\mathrm{NO}_{3}^{-}$do solo Podzolizado de Lins e Marília, var. Marília nos tres períodos de incubação

\section{Efeito do "AM" no Podzolizado de Piracicaba}

O efeito do "AM" neste solo foi significativo aos 60 dias de incubação. O tratamento de $60 \mathrm{ppm}$ de "AM" contribuiu para um maior teor de $\mathrm{NH}_{4}{ }^{+}$e $\mathrm{N}$ total. $\mathrm{O}$ teor de $\mathrm{NO}_{3}{ }^{-}$foi menor em todos os tratamentos com inibidor, indicando efeito do "AM" na nitrificação do $\mathrm{NH}^{4+}$ presente no solo. 


\section{A dose de 60 ppm foi a mais efetiva, mesmo aos 60 dias de incubação.}

Os dados deste solo estão no Quadro 7 e Figuras 7 e 8.

Quadro 7 - Efeito dos tratamentos nos teores de $\mathrm{NH}_{4}^{+} \mathrm{e} \mathrm{NO}_{3}^{-}$do solo Podzolizado de Piracicaba, nos 3 períodos de incubação.

\begin{tabular}{|c|c|c|c|c|c|c|c|c|}
\hline & Tempo & 1 ('Test) & 2 & 3 & 4 & 5 & 6 & 7 \\
\hline \multirow{2}{*}{$15 d$} & $\mathrm{NH}^{+}{ }_{4}$ & 32,20 & 36,40 & 35,58 & 34,88 & 32,90 & 35,70 & 38,03 \\
\hline & $\mathrm{NO}^{-}{ }_{3}$ & $10,50 \mathrm{ab}$ & $8,40 \mathrm{c}$ & $9,19 b c$ & $10,38 \mathrm{ab}$ & $10,85 \mathrm{ab}$ & $10,38 \mathrm{ab}$ & $11,20 \mathrm{a}$ \\
\hline \multirow{2}{*}{$30 \mathrm{~d}$} & $\mathrm{NH}^{+}{ }_{4}$ & 40,13 & 42,70 & 43,63 & 42,58 & 42,58 & 43,40 & 47,25 \\
\hline & $\mathrm{NO}^{-}{ }_{3}$ & $11,32 \mathrm{a}$ & $11,78 \mathrm{a}$ & $11,90 \mathrm{a}$ & $9,10 \mathrm{~b}$ & $8,52 \mathrm{~b}$ & $9,57 \mathrm{~b}$ & $8,52 \mathrm{~b}$ \\
\hline \multirow{2}{*}{$60 \mathrm{~d}$} & $\mathrm{NH}_{4}^{+}$ & $38,03 \mathrm{~cd}$ & $37,22 \mathrm{~d}$ & $39,78 \mathrm{bcd}$ & $42,93 \mathrm{~b}$ & $41,65 b c$ & $45,50 \mathrm{a}$ & $48,07 \mathrm{a}$ \\
\hline & $\mathrm{NO}_{3}{ }_{3}$ & $16,22 \mathrm{a}$ & $13,18 \mathrm{~b}$ & $13,07 \mathrm{~b}$ & $12,60 \mathrm{~b}$ & $13,42 \mathrm{a}$ & $12.72 \mathrm{~b}$ & $13,42 \mathrm{~b}$ \\
\hline
\end{tabular}

Teste $t$ : tratamentos com letras semelhantes não diferem significativamente $(\mathrm{P}<5 \%)$, para cada período de incubação; tratamentos sem letras não diferem significativamente entre si.

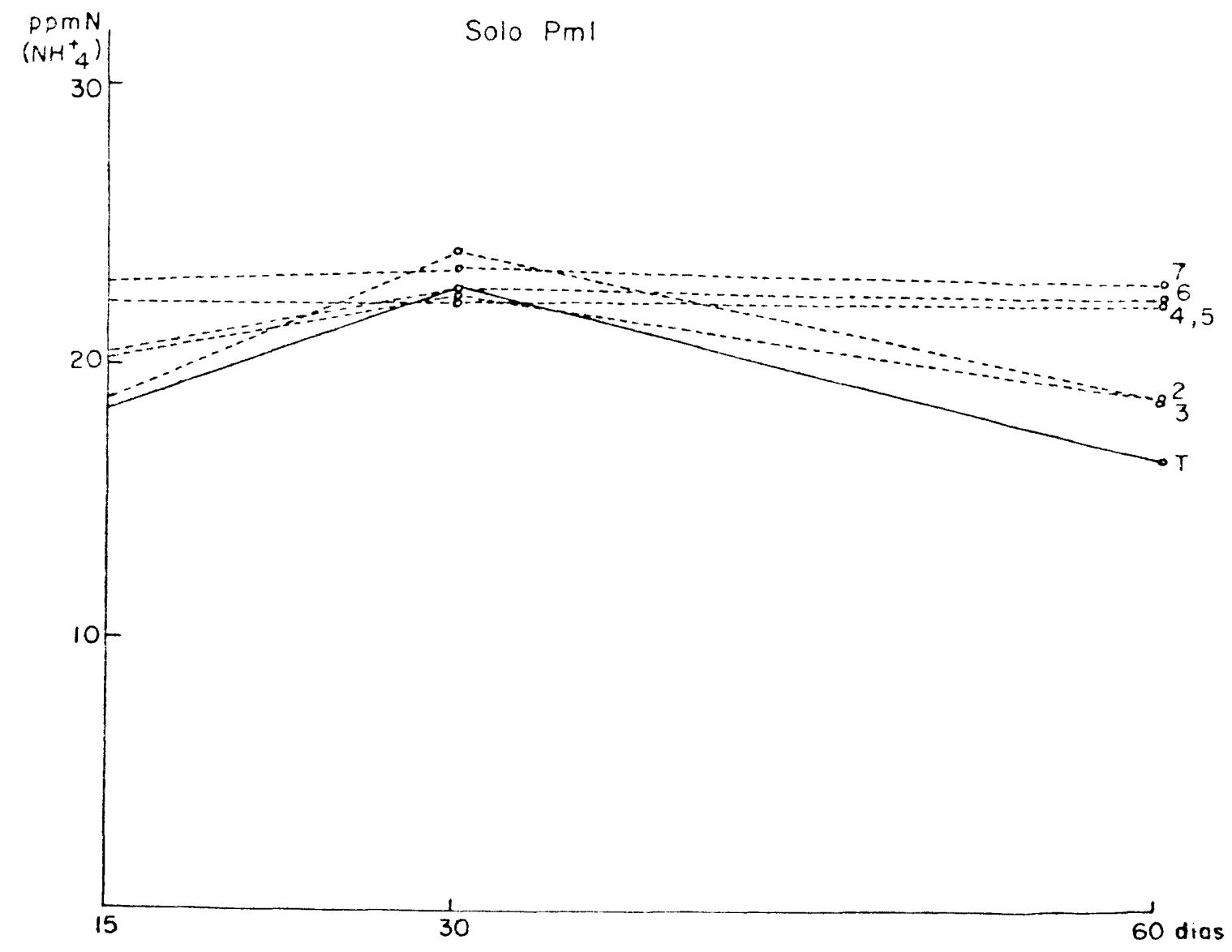

Fig. 7 - Teores de $\mathrm{NH}^{+}{ }_{4}$ do solo Podzolizado de Piracicaba nos tres períodos de incubação 


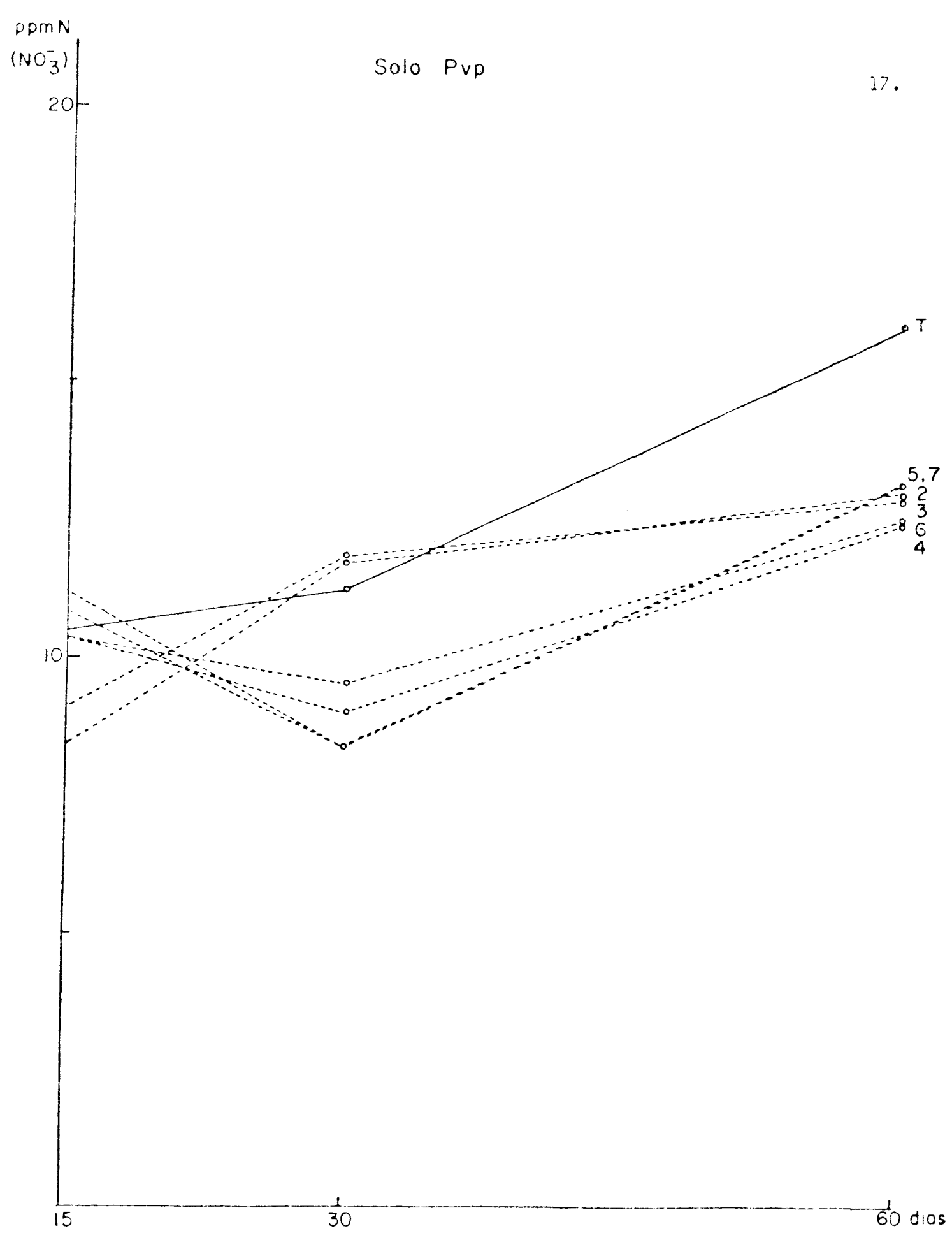

Fig. 8 - Teores de $\mathrm{NO}^{-}$do solo Podzolizado de Piracicaba nos tres períodos de incubação

\section{CONCLUSÕES}

As doses de "AM" não foram suficientes para inibir significativamente a nitrificação no Latossol Roxo e teve pouco efeito no Podzolizado de Lins e Marília, var. Marília. Porém, foram suficientes para atuarem nos solos Podzolizado de Piracicaba e Regossol Intergrade, pelo período de 60 dias, sendo mais efetivas as doses mais elevadas de 48 e 60 ppm 
para o primeiro solo e a dose de $24 \mathrm{ppm}$ para o segundo, já foi suficiente para inibir significativamente a nitrificação.

Para os dois primeiros solos recomendamos posteriores estudos com doses mais elevadas que as utilizadas neste experimento, tomando-se o cuidado de observar a fitotoxidade do produto.

\author{
SUMMARY
}

EFFECT OF THE NITRIFICATION INHIBITOR “AM" IN SOME SOILS OF SÃO PALILO STATE

The nitrification inhibitor "AM" was tested in four different soils of São Paulo State, in order to determine the effective dose for nitrification inhibition for each soil. The doses of "AM" used $(0,12,24,36,48$ and $60 \mathrm{ppm})$ were not sufficient to act significantly in the Latossol Roxo and Podzolizado of Lins and Marília, var. Marília soils; however, positive results were obtained in the Podzolizato of Piracicaba and Regossol Intergrade soils. For the first soil, doses of 48 to $60 \mathrm{ppm}$ were sufficient to act for a period of 60 days and for the second, the "AM" was effective with 24 ppm for a same period of time.

\title{
LITERATURA CITADA
}

BREMnER, J.M., 1965. Total Nitrogen. in: Methorls of Soil Analysis. Part 2: 771-1149, n. ${ }^{\circ}$ 9, Agronomy series. American Society of Agronomy, Inc.

DAS, U.K., 1965. A study on efficiency of utilizing of nitrogen from different sources by hibrid maize and losses of nitrogen from soil during growth period of the crop. M.Sc. Thesis, Division of Agronomy, IARI, New Delhi.

GASSER, J.K. R., GREENLAND, D.J. e RAWSON, R.A.G., 1967. Measurement of losses from fertilizer $\mathrm{N}$ during incubation in acid sandy soils and during subsequent growth of rye-grass, using ${ }^{15} \mathrm{~N}-$ labelled nitrogen. J. Soil Sci., 18:289-300.

GASSER, J.K.R. e IORDANOU, I.G., 1967. Effects of ammonium sulphate and calcium nitrate on the growth, yield and nitrogen uptake of barley, wheat and oats. J. Agric. Sci. Camb., 68: 307-316.

I.AKHADIVE, V.A. e PRASAD, R., 1970. Yield of tall and dwarf indica rice at affected by fertilizer nitrogen, with and without nitrification inhibitors. J. Agric. Sci., Camb. $75375-379$.

PATRICK, W.M.H., PETERSON, F.I. e TURNER, F.T., 1968. Nitrification inhibitors for lowland rice. Soil Sci., 105 : 103-105.

PRASAD, R., 1968. Dry-matter production and recovery of fertilizer nitrogen by rice as affected by nitrification retarders "N-Serve" and "AM". Plant and Soil, 29:327-330. 
PRASAD, R., TURKHEDE, B.B. e DAS, U.K., 1966. 2-chloro-6-(trichloromethyl) pyridine for reducing nitrogen losses from upland paddy soils. Current Science, $35: 312-313$.

RAJALE, G.B. e PRASAD, R., 1970. Nitrification/mineralization of urea as affected by nitrification retarders "N-Serve" and "AM". Current Science, 39:211-212.

WEIR, C.C. e DAVIDSON, J.G., 1968. The effect of retarding nitrification of added fertilizer nitrogen on the yield and $\mathrm{N}$ uptake of Pangola grass. Trop. Agriculture, Trin. 45(4): 301-306.

WEIR, C.C., 1969. The fate of 2-amino-4-chloro-6-methyl pyrimidine (Nitrification Inhibitor) in soil. Trop. Agriculture, Trin., 46:233-238. 
